\title{
THE CONSERVATION EQUATIONS FOR INDEPENDENT COEXISTENT CONTINUA AND FOR MULTICOMPONENT REACTING GAS MIXTURES*
}

\author{
BY \\ W. NACHBAR (Lockhead Aircraft Corporation, Missile Systems Division, Palo Alto, Cal. \\ AND
}

F. WILLIAMS AND S. S. PENNER (California Institute of Technology)

Summary. The equations for conservation of mass, momentum, and energy are derived for a set of independent, coexistent continua obeying the laws of dynamics and thermodynamics. The idea of a control volume and a control surface for each continuum is used in the analysis. The derived results are practically identical with relations obtained previously by Th. von Kármán.

A direct comparison is conducted between the continuum theory results and those obtained from kinetic theory by assuming that, for each of the species, the kinetic theory definitions apply. It is found that the new terms appearing in the conservation equations derived from continuum theory are precisely those which are required to make these equations identical with the results obtained from the kinetic theory of multicomponent, reacting gas mixtures. However, the continuum theory forms of the equations are not useful because they require knowledge of the transport properties for individual species in the mixture.

I. Introduction. The equations for conservation of mass, momentum, and energy for a one-component continuum are well known and are derived in standard treatises on fluid mechanics [1-3]. On the other hand, the conservation equations for reacting, multicomponent, gas mixtures are generally obtained as the equations of change for the summational invariants arising in the solution of the Boltzmann equation $[4,5]$. One of several exceptions to the last statement is the analysis of von Kármán [6] whose results are quoted in a recently published book [7, 8]. Since von Kármán's method of analysis has not been described in detail, and since his results seem to differ from the classical relations through the occurrence of higher order terms in the diffusion velocities, it appeared worthwhile to re-examine this problem with some care.

The objective of our investigation is the derivation of the conservation laws for multicomponent, reacting, gas mixtures. To this end we invent a physical model consistent with continuum theory. Our model involves the idea of a multicomponent continuum composed of coexistent continua, each obeying the laws of dynamics and thermodynamics, a notion which was first introduced by Stefan in $1871 \uparrow$. For an $n$-component gas mixture we presume the existence of $n$ distinct continua within any arbitrary volume, continuum $K$ corresponding to the chemical species $K$. We shall use the terms continuum $K$, species $K$, and component $K$ interchangeably, it being understood that each of these phrases refers to continuum $K$ of the coexistent continua as long as we are following

\footnotetext{
*Received December 13, 1957. This research was supported by the United States Air Force through the Air Force Office of Scientific Research of the Air Research and Development Command under Contract AF 18(603)-146.

$\dagger$ We arrive at the model of simultaneous coexistent continua as the logical transcription to continuum theory of the fact that the entire volume is accessible to all of the different molecules in a gas mixture.
} 
the derivation of conservation laws from continuum theory. It is apparent that each space point in the multicomponent continuum has $n$ velocities $v_{i}^{K}(K=1,2, \cdots, n)$, one velocity for each of the coexistent continua.

In Sec. II we present relevant definitions and basic mathematical relations, which will be used in subsequent sections. In Secs. III to $\mathrm{V}$ we treat, respectively, the equations for conservation of mass, momentum, and energy. Our results are considered critically in Sec. VI and are compared with the relations obtained from the kinetic theory of non-uniform gas mixtures.

II. Definitions and basic mathematical relations. The multicomponent continuum is considered to be defined in regions of space, every point in a region being an interior point of the region. All properties of the $n$ continua, including the velocities $v_{i}^{K}(K=1,2, \cdots, n)$, are assumed to be described by functions continuously differentiable in all variables within the region. This statement will be said to define "continuous flow" for the multicomponent continuum.

The conservation equations for continuous flow of species $K$ will be derived by using the idea of a control volume $\tau^{K}(t)$ enclosed by its control surface $\sigma^{K}(t)$, and lying wholly within a region occupied by the continuum; here " $t$ " denotes the time. The notation of cartesian tensors will be used*. Let $x_{i}(i=1,2,3)$ denote the cartesian coordinates of a point in space. In cartesian tensor notation, the divergence theorem for any scalar function belonging to the $K$ th continuum $\alpha^{K}\left(x_{i}, t\right)$, becomes

$$
\int_{\sigma K} \alpha^{K} n_{i}^{K} d \sigma=\int_{\tau} \alpha_{, j}^{K} d \tau,
$$

where $n_{i}^{K}$ denotes the outward normal to the surface $\sigma^{K}$ and $\alpha_{i}^{K}$ represents the gradient of the scalar $\alpha^{K}$. For any vector function belonging to the $K$ th continuum, $u_{i}^{K}\left(x_{\imath}, t\right)$, we have

$$
\int_{\sigma} u_{i}^{K} n_{i}^{K} d \sigma=\int_{\tau^{K}} u_{i, i}^{K} d \tau,
$$

with $u_{i, j}^{R}$ denoting the divergence of the vector $u_{i}^{R}$.

Consider that some property of the $K$ th continuum has a density per unit volume equal to $\alpha^{K}\left(x_{i}, t\right)$, and let $A^{K}(t)$ be the amount of this property contained within the control volume $\tau^{K}$. Thus

$$
A^{K}(t)=\int_{\tau} \alpha^{K}\left(x_{i}, t\right) d \tau .
$$

For example, if $\alpha^{K}=\rho^{K}=$ the density of mass of species $K$, then $A^{K}$ is the total mass of species $K$ contained within $\tau^{K}$. The property $\alpha^{K}$ has a density per unit mass of mixture equal to $\beta^{R}\left(x_{i}, t\right)$ where

$$
\alpha^{K}=\rho \beta^{K},
$$

with $\rho=\sum_{K=1}^{n} \rho^{K}$ representing the density of mass for the fluid mixture.

The derivative $d A^{K} / d t$ is defined to mean the time rate of change of $A^{K}$ as the volume $\tau^{K}$ and its surface $\sigma^{K}$ move with the flow of species $K$. Consider that Eq. (3) holds at a time $t_{0}$; at time $t_{0}+\Delta t$ the particles in $\tau^{K}$ at $x_{i}$ will have been displaced to new positions

*Repeated subscript indices imply summation over all allowed values of the indices. 
$x_{i}^{\prime}$ and will be contained within some new volume $\tau^{K}\left(t_{0}+\Delta t\right)=\tau^{\prime K}$ enclosed by a surface $\sigma^{K}\left(t_{0}+\Delta t\right)={\sigma^{\prime}}^{K}$; in general ${\tau^{\prime K}}^{K}$ and ${\sigma^{\prime}}^{K}$ are different from $\tau^{K}$ and $\sigma^{K}$. Therefore

$$
A^{K}\left(t_{0}+\Delta t\right)=\int_{\tau^{\prime} K} \alpha^{K}\left(x_{i}^{\prime}, t_{0}+\Delta t\right) d \tau
$$

and

$$
\left(\frac{d A^{K}}{d t}\right)_{t=t_{0}}=\lim _{\Delta t \rightarrow 0}\left\{\frac{1}{\Delta t}\left[\int_{\tau^{\prime} K} \alpha^{K}\left(x_{i}^{\prime}, t_{0}+\Delta t\right) d \tau-\int_{\tau^{K}} \alpha^{K}\left(x_{i}, t_{0}\right) d \tau\right]\right\} .
$$

It is demonstrated in the appendix that Eq. (5) is equivalent to the relation

$$
\frac{d A^{K}}{d t}=\int_{\tau^{K}}\left[\frac{\partial \alpha^{K}}{\partial t}+\left(\alpha^{K} v_{i}^{K}\right)_{, i}\right] d \tau
$$

Hence, using the divergence theorem given in Eq. (2), it is fouud that

$$
\frac{d A^{K}}{d t}=\int_{\tau^{K}} \frac{\partial \alpha^{K}}{d t} d \tau+\int_{\sigma^{K}} \alpha^{K} v_{i}^{K} n_{i} d \sigma .
$$

Equation (7) expresses the idea that the time rate of change of $A^{K}$ in a flow, for an arbitrary volume $\tau^{K}$ bounded by a surface $\sigma^{K}$, is equal to the stationary rate of change of $A^{R}$ in the interior of $\tau^{K}$ plus the rate of change of $A^{K}$ due to the movement of $\tau^{K}$ and $\sigma^{K}$.

Equation (6) may be rewritten in the equivalent form

$$
\frac{d}{d t}\left(\int_{\tau^{K}} \rho \beta^{K} d \tau\right)=\int_{\tau^{K}}\left[\frac{\partial\left(\rho \beta^{K}\right)}{\partial t}+\left(\rho \beta^{K} v_{i}^{K}\right)_{, i}\right] d \tau .
$$

The overall transport equation for the multicomponent continuum is then obtained by summing over components, a procedure which is in accord with the idea of independent coexistent continua. We choose at the arbitrary time $t$ all of the control volumes $\tau^{K}$ to be coexistent, i.e., $\tau^{K} \equiv \tau$ for all $K$. We will henceforth refer to a volume $\tau$ thus defined as being "of the multicomponent continuum" at time $t$. After summation, Eq. (8) now becomes

$$
\sum_{K}\left(\frac{d}{d t} \int_{\tau^{K}} \rho \beta^{K} d \tau\right)_{\tau^{K}=\tau}=\int_{\tau}\left[\frac{\partial}{\partial t}\left(\sum_{K} \rho \beta^{K}\right)+\sum_{K}\left(\rho \beta^{K} v_{i}^{K}\right)_{, i}\right] d \tau .
$$

III. Continuity equations. We denote by $w^{K}$ the net production of mass of species $K$ per unit volume per unit time. Since mass is not created or destroyed by chemical reactions, but only converted from one species to another, it follows that

$$
\sum_{K} w^{K}=0 .
$$

The continuity of the mass of species $K$ in an arbitrary volume $\tau^{K}$ is therefore expressed by the equation

$$
\frac{d}{d t}\left(\int_{\tau^{K}} \rho Y^{K} d \tau\right)=\int_{\tau^{K}} w^{K} d \tau,
$$

where $Y^{K}$ is the mass or weight fraction of species $K$ (i.e., $\rho^{K}=\rho Y^{K}$ and $Y^{K}$ equals the mass of species $K$ in unit mass of mixture). Let $\beta^{K}=Y^{K}$ in Eq. (8); then Eq. (11) becomes

$$
\int_{\tau^{K}}\left[\frac{\partial\left(\rho Y^{K}\right)}{\partial t}+\left(\rho Y^{K} v_{i}^{K}\right)_{, i}-w^{K}\right] d \tau=0
$$


and, since $\tau^{K}$ is arbitrary, we have

$$
w^{K}=\frac{\partial\left(\rho Y^{K}\right)}{\partial t}+\left(\rho Y^{K} v_{i, i}^{K}\right.
$$

Now let $v_{i}^{K}$, the flow velocity for species $K$, be represented as

$$
v_{i}^{K}=v_{i}^{\prime}+V_{i}^{K},
$$

where

$$
v_{i}^{\prime} \equiv \sum_{K} Y^{K} v_{i}^{K}
$$

The summation in Eq. (14) is extended over all $n$ distinct chemical components. Thus $v_{i}^{\prime}$ is the mass-weighted average velocity of the fluid mixture, and $V_{i}^{K}$ is said to denote the diffusion velocity of species $K$. Since

$$
\sum_{K} Y^{K}=1
$$

it follows from Eqs. (13) and (14) that

$$
\sum_{K} Y^{K} V_{i}^{K}=0
$$

Introducing Eq. (13) into Eq. (12) leads to the following equation for continuity of species $K$ :

$$
w^{K}=\frac{D}{D t}\left(\rho Y^{K}\right)+\rho Y^{K} v_{i, i}^{\prime}+\left(\rho Y^{K} V_{i, i}^{K},\right.
$$

where

$$
\frac{D}{D t}() \equiv \frac{\partial}{\partial t}()+v_{i}^{\prime}()_{, i}
$$

is the Euler total time derivative following the mass-weighted average motion of the multicomponent continuum. Summing Eq. (17) over all distinct components, in view of Eqs. (10), (15) and (16), leads to the overall continuity equation

$$
\frac{D \rho}{D t}+\rho v_{i, i}^{\prime}=0 \text {. }
$$

Equation (19) is evidently also the correct form of the continuity equation for a onecomponent system.

We may now transform Eq. (9) by using Eqs. (18) and (19) to obtain a form which is useful for the derivation of the differential equations expressing conservation of momentum and energy, viz.,

$$
\sum_{K}\left(\frac{d}{d t} \int_{\tau K} \rho \beta^{K} d \tau\right)_{\tau, K=\tau}=\int_{\tau}\left[\rho \frac{D \beta}{D t}+\left(\rho \sum_{K} \beta^{K} V_{i}\right)_{, i}\right] d \tau,
$$

where

$$
\beta \equiv \sum_{K} \beta^{K}
$$


IV. Momentum equations. For an arbitrary volume $\tau$ of the multicomponent continuum, the total rate of change of linear momentum in the $j$ th coordinate direction must equal the sum of the following: (a) the surface integral of the stress vector $\sum_{K} \sigma_{i i}^{K} n_{i}$, where $\sigma_{i j}^{K}$ equals* the component in the direction $x_{i}$ of the stress vector acting on that face of an elemental parallelepiped of species $K$ which has outward normal in the direction $x_{i}$; (b) the volume integral of the total vector body force $\sum_{K} \rho^{K} f_{i}^{K}$ acting on unit volume of mixture, where $f_{i}^{K}$ is the vector body force per unit mass of species $K$; and (c) the volume integral of the total rate of generation of momentum in unit volume through production of species. Let the rate of generation of momentum in unit volume for species $K$ be $w^{K} m_{i}^{K}$, where $m_{i}^{K}$ is the average momentum of the generated mass of species $K$ per unit mass of species $K$. We postulate that, overall, linear momentum is neither created nor destroyed by chemical reactions; the consequent conservation principle states that the total rate of generation of linear momentum per unit volume by chemical production of species is zero:

$$
\sum_{K} w^{K} m_{i}^{K}=0 .
$$

The total rate of change of linear momentum is then expressed mathematically by

$$
\sum_{K}\left(\frac{d}{d t} \int_{\tau^{K}} \rho Y^{K} v_{i}^{K} d \tau\right)_{\tau^{K}=\tau}=\int_{\sigma} \sum_{K} \sigma_{i j}^{K} n_{i} d \sigma+\int_{\tau} \sum_{K} \rho^{K} f_{i}^{K} d \tau .
$$

Using the divergence theorem, Eq. (1), and the transport relation given in Eq. (20), with

$$
\begin{gathered}
\beta^{K}=Y^{K} v_{i}^{K}, \\
\beta=v_{i}^{\prime},
\end{gathered}
$$

Eq. (2) becomes

$$
\int_{\tau}\left[\rho \frac{D v_{i}^{\prime}}{D t}+\left(\rho \sum_{K} Y^{K} V_{i}^{K} V_{i}^{K}\right)_{, i}\right] d \tau=\int_{\tau} \sum_{K}\left(\sigma_{i, i}^{K}+\rho^{K} f_{i}^{K}\right) d \tau .
$$

We now define $\sigma_{i i}^{D}$, the diffusion stress tensor, as

$$
\sigma_{i j}^{D} \equiv-\rho \sum_{K} Y^{K} V_{i}^{K} V_{i}^{K}
$$

and $f_{i}$, the vector body force per unit mass of mixture, as

$$
f_{i}=\sum_{K} f_{i}^{K} Y^{K} .
$$

Since $\tau$ is arbitrary, Eq. (23) then leads to an expression for overall conservation of momentum, namely,

$$
\rho \frac{D v_{i}^{\prime}}{D t} \equiv \rho \frac{\partial v_{i}^{\prime}}{\partial t}+\rho v_{i}^{\prime} v_{i, i}^{\prime}=\sum_{K} \sigma_{i, i}^{K}+\sigma_{i i, i}^{D}+\rho f_{i} .
$$

If we define $\sigma_{i j}$ as

$$
\sigma_{i j} \equiv \sum_{K} \sigma_{i j}^{K}+\sigma_{i i}^{D}
$$

${ }^{*}$ The species vectors $\sigma_{i j}^{K} n_{i}$ and $\rho f_{j}^{K}$ represent the sums of all forces which act upon species $K$ and which move with the velocity of species $K$ in the mixture. These definitions are used in Sec. VI to identify our results with the results obtained from kinetic theory. 
then Eq. (26) is reduced to the well known form of the momentum equation for onecomponent systems. Therefore, $\sigma_{i j}$ is the stress tensor and $\rho f_{i}$ is the body force acting on an elemental parallelepiped which is moving with the mass-weighted average velocity $v_{i}^{\prime}$. Furthermore, we can express $\sigma_{i i}$ as a sum of partial stress tensors $\sigma_{i j}^{*},{ }^{k}$

$$
\sigma_{i i} \equiv \sum_{K} \sigma_{i i}^{*},{ }^{*}
$$

where, from Eqs. (24) and (27)

$$
\sigma_{i j}^{*}{ }^{K} \equiv \sigma_{i j}^{K}-\rho Y^{K} V_{i}^{K} V_{i}^{K} .
$$

Each stress tensor can then always be expressed as the sum of a mean pressure tensor, a viscous stress tensor, and a viscous diffusion stress tensor; thus,

$$
\begin{aligned}
\sigma_{i j}^{*}, K & \equiv-p^{K} \delta_{i j}+\tau_{i j}^{V, K}+\tau_{i j}^{D, K}, & \text { where } & p^{K} \equiv-\frac{1}{3} \sigma_{i i}^{*}, \\
\sigma_{i j} & \equiv-p \delta_{i j}+\tau_{i j}^{\nabla}+\tau_{i j}^{D}, & & \text { where } \quad p \equiv-\frac{1}{3} \sigma_{i i} .
\end{aligned}
$$

The total pressure $p$ is the sum of the partial pressures $p^{K}$ for the different species, i.e.,

$$
p=\sum_{K} p^{K}
$$

and so, in view of Eq. (28), it follows now that

$$
\begin{aligned}
\tau_{i j}^{\nabla} & =\sum_{K} \tau_{i i}^{\nabla, K}, \\
\tau_{i j}^{D} & =\sum_{K} \tau_{i i}^{D, K} .
\end{aligned}
$$

The equations of von Kármán [6, 7] are obtained by using Eq. (30) in Eq. (26), viz.,

$$
\rho \frac{D v_{i}^{\prime}}{D t}=-p_{. j}+\left(\tau_{i j}^{V}+\tau_{i j}^{D}\right)_{, i}+\rho f_{i} .
$$

V. Energy equation. For an arbitrary volume $\tau$ of the multicomponent continuum, the first law of thermodynamics states that:

Rate of increase of (internal plus kinetic energy) $=$ rate at which work is done on $\tau$ by (body forces plus surface stresses) + rate of inward transport of heat by thermal conduction through the surface $\sigma$ enclosing $\tau+$ rate of generation of energy through production of species within $\tau+$ rate at which work is done on material produced within $\tau$.

Let $u^{K}$ denote the absolute internal energy of species $K$ per unit mass of species $K$ and let $u$ denote the absolute internal energy per unit mass of mixture. Then

$$
u \equiv \sum_{K} Y^{K} u^{K} \text {. }
$$

The kinetic energy of species $K$ per unit mass of species $K$ is $\frac{1}{2} v_{i}^{K} v_{i}^{K}$. The total rate at which work is done on $\tau$ by surface stresses and body forces is represented as the superposition of the rate of work done on the individual continua by their own surface stresses and body forces.

For the mass $w^{K}$ of species $K$, which is generated by chemical reaction in unit volume per unit time, the sum of (a) the internal and kinetic energy carried by this mass, and (b) the work done on this mass in unit time, is $w^{K}\left(\eta^{K}+\frac{1}{2} m_{i}^{K} m_{i}^{K}\right)$, where $\eta^{K}$ is the average 
specific enthalpy of generated mass of species $K^{*}$. We postulate that, overall, energy is redistributed among various states but is neither created nor destroyed by chemical reaction; the consequent conservation principle states that the total rate of generation of (absolute enthalpy plus kinetic energy) per unit volume by chemical production of species is zero:

$$
\sum_{K} w^{K}\left(\eta^{K}+\frac{1}{2} m_{i}^{K} m_{i}^{K}\right)=0 .
$$

The analytical expression of the first law of thermodynamics, subject to the fundamental postulates of independence and conservation, is therefore

$$
\begin{aligned}
\sum_{K}\left[\frac{d}{d t} \int_{\tau} \rho Y^{K}\left(u^{K}+\frac{1}{2} v_{i}^{K} v_{i}^{K}\right) d \tau\right]_{\tau} K_{-\tau} \\
=\sum_{K}\left[\int_{\sigma} \sigma_{i i}^{K} n_{i} v_{i}^{K} d \sigma+\int_{\tau} \rho f_{i}^{K} v_{i}^{K} d \tau\right]-\int_{\sigma} \sum_{K} q_{i}^{K} n_{i} d \sigma .
\end{aligned}
$$

Here $q_{i}$ is the heat flux vector for species $K$, taken as positive for outwards heat transport. Equation (37) can be transformed by the use of Eq. (21) with

$$
\beta^{K}=Y^{K} u^{K}+\frac{1}{2} Y^{K} v_{i}^{K} v_{i}^{K}
$$

and

$$
\beta=u+\frac{1}{2} v_{i}^{\prime} v_{i}^{\prime}+\frac{1}{2} \sum_{K} Y^{K} V_{i}^{K} V_{i}^{K} .
$$

Since $\tau$ is arbitrary, the following differential equation for overall conservation of energy then results:

$$
\begin{array}{r}
\rho \frac{D}{D t}\left(u+\frac{1}{2} v_{j}^{\prime} v_{i}^{\prime}\right)+\frac{1}{2} \rho \frac{D}{D t}\left(\sum_{K} Y^{K} V_{i}^{K} V_{i}^{K}\right)+\left[\rho \sum_{K}\left(Y^{K} u^{K} V_{i}^{K}+\frac{1}{2} Y^{K} v_{i}^{K} v_{i}^{K} V_{i}^{K}\right)\right]_{, i} \\
=\rho \sum_{K} Y^{K} f_{i}^{K} v_{i}^{K}+\sum_{K}\left(\sigma_{i ;}^{K} v_{i}^{K}\right)_{, i}-\sum_{K} q_{i, i}^{K} .
\end{array}
$$

Let $h^{K}$ denote the absolute specific enthalpy of species $K$, which is defined as

$$
Y^{K} h^{K}=Y^{K} u^{K}+\frac{p^{K}}{\rho}
$$

The absolute specific enthalpy of the mixture is then $h=\sum_{K} Y^{K} h^{K}=u+p / \rho$. The total heat flux vector is assumed to be expressible in the form

$$
\sum_{K} q_{i}^{K}=-\lambda T_{, i}
$$

where $T$ is the temperature, and $\lambda$ is the thermal conductivity. Using the definitions given by Eqs. (29), (30), (40) and (41), Eq. (39) can be written in a desired form, viz.,

$$
\begin{aligned}
\rho \frac{D}{D t}\left(u+\frac{1}{2} v_{j}^{\prime} v_{j}^{\prime}\right)+\left(\rho \sum_{K} Y^{K} h^{K} V_{i}^{K}\right)_{, i}=\rho f_{i} v_{i}^{\prime}-\left(p v_{i}^{\prime}\right)_{, i}+\left[\left(\tau_{i i}^{V}+\tau_{i j}^{D}\right) v_{i}^{\prime}\right]_{, i} \\
+\left(\lambda T_{, i}\right)_{, i}+\rho \sum_{K} Y^{K} V_{i}^{K} f_{i}^{K}+\sum_{K}\left[\left(\tau_{i j}^{V, K}+\tau_{i j}^{D, K}\right) V_{i, i}^{K}\right]_{, i} \\
+\frac{1}{2}\left(\rho \sum Y^{K} V_{i}^{K} V_{i}^{K} V_{i}^{K}\right)_{, i}-\rho \frac{D}{D t}\left[\frac{1}{2} \sum_{K} Y^{K} V_{i}^{K} V_{i}^{K}\right] .
\end{aligned}
$$

*The quantity $\eta^{K}$ should not be confused with the total (average) specific enthalpy of species $K$ which we denote by $h^{K}$, as in Eq. (40). 
Eq. (42) is practically identical with the energy equation derived by von Kármán [6, 7]. When the diffusion velocities vanish, Eq. (42) reduces to a well-known form of the energy equation for one-component systems.

VI. Comparison between the conservation laws derived for independent coexistent continua and the kinetic theory results for multicomponent gas mixtures. In order to show that the model of independent, coexistent continua represents correctly a real mixture of gases composed of different chemical species, we must compare the results obtained from this model with those of the kinetic theory of non-uniform gas mixtures. Quantities such as the density $\rho$, the mass-weighted average velocity $v_{i}^{\prime}$, and body force $f_{1}$, have obviously analogous meanings in both the kinetic theory and the coexistent continua model. On the other hand, the precise kinetic-theory meaning of terms such as the stress tensor $\sigma_{i j}^{K}$, the absolute internal energy per unit mass $u^{K}$, and the heat flux vector $q_{i}^{K}$ is not immediately apparent. In view of the known success of continuum theory for one-component systems, we shall identify the continuum theory properties $\sigma_{i i}^{K}, u^{K}$, and $q_{i}^{K}$ for species $K$ with their kinetic theory counterparts. Our proof then involves a comparison between the conservation equations obtained from multicomponent continuum theory, replacing continuum properties for each species by their kinetic theory definitions, and the conservation equations obtained from the kinetic theory of non-uniform gas mixtures.

$A$. Definitions of kinetic theory. Let $c_{i}^{K, m}$ be the velocity of a particular molecule, $m$, of species $K$, and let $V_{i}^{\prime K, m}$ be the velocity of this molecule in excess of the velocity $v_{i}^{R}$, which is identified in kinetic theory as the mean velocity of all molecules of species $K$. Then

$$
c_{i}^{K, m}=v_{i}^{K}+V_{i}^{\prime K, m} \equiv v_{i}^{\prime}+V_{i}^{K}+V_{i}^{\prime K, m}, \quad\left\langle c_{i}^{K, m}\right\rangle=v_{i}^{K} \quad \text { and } \quad\left\langle V_{i}^{\prime K, m}\right\rangle=0,
$$

where the angle brackets indicate an average over all molecules of species $K$ taken with respect to a distribution function appropriate for the mixture.

From kinetic theory, we have the following definitions ${ }^{1}$ for the properties of species $K$ in the mixture

$$
\begin{gathered}
\sigma_{i i}^{K} \equiv-\rho^{K}\left\langle V_{i}^{\prime K, m} V_{i}^{\prime K, m}\right\rangle \\
u^{K} \equiv\left\langle\frac{1}{2} V_{i}^{\prime K, m} V_{i}^{\prime K, m}+i^{K, m}\right\rangle \equiv \frac{1}{2}\left\langle V_{i}^{\prime K, m} V_{i}^{\prime K, m}\right\rangle+i^{K} \\
q_{i}^{K} \equiv \rho^{K}\left\langle\left(\frac{1}{2} V_{i}^{\prime K, m} V_{i}^{\prime K, m}+i^{K, m}\right) V_{i}^{\prime K, m}\right\rangle \equiv \rho^{K}\left(\left\langle\frac{1}{2} V_{i}^{\prime K, m} V_{i}^{\prime K, m} V_{i}^{\prime K, m}\right\rangle+\left\langle i^{K, m} V_{i}^{\prime K, m}\right\rangle\right) .
\end{gathered}
$$

In Eqs. (44) and (45), $u^{K, m}$, the total internal energy per unit mass of a molecule of species $K$, is expressed as the sum of $\left(\frac{1}{2} V_{i}^{\prime K, m} V_{i}^{\prime K, m}\right)$, the peculiar translatory kinetic energy per unit mass, and $\left(i^{K, m}\right)$, the contribution of additional internal energy terms (rotational, vibrational, etc.) per unit mass. We have then defined $u^{K} \equiv\left\langle u^{K, m}\right\rangle$ and $i^{K} \equiv\left\langle i^{K, m}\right\rangle$.

The corresponding definitions in the kinetic theory for the properties of the gas mixture will be denoted by the superscript $T$; these $\operatorname{are}^{2}$ : the mixture stress tensor $\sigma_{i j}^{T}$, where 


$$
\begin{aligned}
\sigma_{i j}^{T} \equiv-\sum_{K} \rho^{K}\left\langle( V _ { i } ^ { \prime K , m } + V _ { i } ^ { K } ) \left( V_{i}^{\prime K, m}\right.\right. & \left.\left.+V_{i}^{K}\right)\right\rangle \\
& \equiv-\sum_{K} \rho^{K}\left\langle V_{i}^{\prime K, m} V_{i}^{\prime K, m}\right\rangle-\sum_{K} \rho^{K} V_{i}^{K} V_{i}^{K},
\end{aligned}
$$

the internal energy per unit mass of mixture $u^{T}$, where

$$
\begin{aligned}
\rho u^{T} \equiv \sum_{K}\left[\frac { 1 } { 2 } \rho ^ { K } \left\langle\left(V_{i}^{\prime K, m}+\right.\right.\right. & \left.\left.\left.V_{i}^{K}\right)\left(V_{i}^{\prime K, m}+V_{i}^{K}\right)\right\rangle+\rho^{K} i^{K}\right] \\
& \equiv \frac{1}{2} \sum_{K} \rho^{K}\left\langle V_{i}^{\prime K, m} V_{i}^{\prime K, m}\right\rangle+\frac{1}{2} \sum_{K} \rho^{K} V_{i}^{K} V_{i}^{K}+\sum_{K} \rho^{K} i^{K}
\end{aligned}
$$

and the heat flux vector for the mixture $q_{i}^{T}$, where

$$
\begin{aligned}
& q_{i}^{T} \equiv \sum_{K} \rho^{K}\left\langle\left[\frac{1}{2}\left(V_{i}^{\prime K, m}+V_{i}^{K}\right)\left(V_{i}^{\prime K, m}+V_{i}^{K}\right)+i^{K, m}\right]\left(V_{i}^{\prime K, m}+V_{i}^{K}\right)\right\rangle \\
& \equiv \sum_{K} \rho^{K}\left\{\left\langle\frac{1}{2} V_{i}^{\prime K, m} V_{i}^{\prime K, m} V_{i}^{\prime K, m}\right\rangle+\right. \frac{1}{2} V_{i}^{K}\left\langle V_{i}^{\prime K, m} V_{i}^{\prime K, m}\right\rangle+V_{i}^{K}\left\langle V_{i}^{\prime K, m} V_{i}^{\prime K, m}\right\rangle \\
&\left.+\frac{1}{2} V_{i}^{K} V_{i}^{K} V_{i}^{K}+\left\langle i^{K, m} V_{i}^{\prime K, m}\right\rangle+i^{K} V_{i}^{K}\right\} .
\end{aligned}
$$

Using Eqs. (43), (44) and (45) in Eqs. (46), (47) and (48), the following identities are obtained between the properties $\sigma_{i i}^{T}, \rho u^{T}$ and $q_{i}^{T}$ of the gas mixture and the properties $\sigma_{i j}^{K}, \rho^{K} u^{K}$ and $q_{i}^{K}$ of the individual species:

$$
\begin{aligned}
\sigma_{i j}^{T} & \equiv \sum_{K} \sigma_{i i}^{K}-\sum_{K} \rho^{K} V_{i}^{K} V_{i}^{K}, \\
\rho u^{T} & \equiv \sum_{K} \rho^{K} u^{K}+\frac{1}{2} \sum_{K} \rho^{K} V_{i}^{K} V_{i}^{K}, \\
q_{i}^{T} & \equiv \sum_{K} q_{i}^{K}-\sum_{K} \sigma_{i j}^{K} V_{i}^{K}+\sum_{K} \rho^{K} u^{K} V_{i}^{K}+\frac{1}{2} \sum_{K} \rho^{K} V_{i}^{K} V_{i}^{K} V_{i}^{K} .
\end{aligned}
$$

In each of the above relations, the property for the mixture is equal to the sum, over all species, of the corresponding property for the components plus various diffusion terms. The diffusion terms arise because the reference coordinate system for species $K$ is taken to move with velocity $v_{i}^{\prime}+V_{i}^{K}$, which is the mass-weighted average velocity for molecules in species $K$ alone; the reference coordinate system for the mixture on the other hand is taken to move with velocity $v_{j}^{\prime}$, which is the mass-weighted average velocity for all molecules in the mixture.

$B$. Comparison of conservation equations. The continuity equation for species $K$, as given by Eq. (17), is readily seen to be identical with the corresponding relation in the kinetic theory for multicomponent gas mixtures if $w^{K}$ is the net mass rate of production of species $K$ per unit volume by chemical reaction ${ }^{3}$. Explicit evaluation of $w^{K}$ requires the introduction of the laws of chemical kinetics.

The expression for overall conservation of momentum, Eq. (26), is likewise identical with the corresponding relation in the kinetic theory ${ }^{5}$, as a comparison of Eqs. (27) and (49) shows that $\sigma_{i j} \equiv \sigma_{i j}{ }^{T}$.

3See, for example, Eq. (4) of Sec. 8.1 in [4]. For chemical reactions, the right hand side of this equation does not vanish but is equal to $w^{K} / m^{K}$, where $m^{K}$ is the mass of a molecule of species $K$.

'See, for example, Chap. I in [7].

${ }^{5}$ See, for example, Eq. (7) of Sec. 8.1 in [4]. 
To demonstrate the equivalence of the energy conservation equations, we rewrite Eq. (39) in the form

$$
\begin{aligned}
\rho \frac{D}{D t}\left[\sum_{K}\left(Y^{K} u^{K}+\frac{1}{2} Y^{K} V_{i}^{K} V_{i}^{K}\right)\right. & \left.+\frac{1}{2} v_{j}^{\prime} v_{i}^{\prime}\right] \\
& +\left(\rho \sum_{K} Y^{K} u^{K} V_{i}^{K}\right)_{, i}=\rho f_{i} v_{i}^{\prime}+\rho \sum_{K} Y^{K} f_{i}^{K} V^{K} \\
& +\left(v_{i}^{\prime} \sum_{K} \sigma_{i j}^{K}\right)_{, i}+\sum_{K}\left(\sigma_{i j}^{K} V_{i}^{K}\right)_{, i}-\sum_{K} q_{i, i}^{K} \\
& -\left(\rho v_{i}^{\prime} \sum_{K} Y^{K} V_{i}^{K} V_{i}^{K}\right)_{, i}-\frac{1}{2}\left(\rho \sum_{K} Y^{K} V_{i}^{K} V_{i}^{K} V_{i}^{K}\right)_{, i} .
\end{aligned}
$$

Replacing $\sum_{K} \sigma_{i i}^{K}, \sum_{K} Y^{K} u^{K}$ and $\sum_{K} q_{i}^{K}$ by their kinetic theory equivalents, as given by Eqs. (49), (50), and (51), Eq. (52) becomes

$$
\rho \frac{D}{D t}\left[u^{T}+\frac{1}{2} v_{j}^{\prime} v_{i}^{\prime}\right]=\rho f_{i} v_{i}^{\prime}+\rho \sum_{K} Y^{K} f_{i}^{K} V_{i}^{K}-q_{i, i}^{T}+\left(\sigma_{i i}^{T} v_{i}^{\prime}\right)_{, i} .
$$

Multiplying the momentum conservation equations by $v_{i}^{\prime}$ and contracting, the following scalar equation is found:

$$
\frac{1}{2} \rho \frac{D}{D t}\left(v_{i}^{\prime} v_{i}^{\prime}\right)=\sigma_{i, i, i}^{T} v_{i}^{\prime}+\rho f_{i} v_{i}^{\prime} .
$$

Using this relation and Eq. (19), Eq. (53) can be placed in a form which is identical with the usual form of the result obtained from kinetic theory ${ }^{6}$, viz.

$$
\frac{D}{D t}\left(\rho u^{T}\right)+\rho u^{T} v_{i, i}^{\prime}=\rho \sum_{K} Y^{K} f_{i}^{K} V_{i}^{K}-q_{i, i}^{T}+\sigma_{i, i}^{T} v_{i, i}^{\prime} .
$$

C. Concluding remarks. We have shown that, with proper identifications, the conservation equations for mass, momentum, and energy which are derived from the model of independent, coexistent continua (multicomponent continuum) are those obeyed by real gas mixtures. This conclusion is not surprising, in view of the fact that the results derived from this model, as well as those obtained from kinetic theory, do not depend on the forces operative in molecular collisions. The total mass, momentum, and energy are conserved in collisions; they are summational invariants. One would expect, in general, that the independent, coexistent continua model will give the correct conservation equations for summational invariants.

However, apart from extending the one-component continuum results in a natural way to the flow of reacting mixtures, the multicomponent continuum model does not lead to any new results which are presently useful. In particular, to express conservation of energy for the mixture, Eq. (42) requires knowledge of species transport terms $u^{K}$, $\sigma_{i i}^{K}$ and $q_{i}^{K}$ in the mixture; these terms cannot be evaluated by kinetic theory methods. The mixture transport terms $u^{T}, \sigma_{i j}^{T}$ and $q_{i}^{T}$ can be evaluated, however, and therefore it is Eq. (55), or an equivalent form, which must be used to express energy conservation for the mixture. 


\section{APPENDIX}

Proof of Eq. (6). In order to prove Eq. (6), it is more convenient to work from Eq. (3) than from the limiting relation given in Eq. (5), and also to introduce the Lagrangian representation [9]. For any continuum $K$, let the three parameters $a_{i}^{K}$ identify the individual point particles of continuum $K$; for definiteness, suppose that $a_{i}^{K}$ are the spatial coordinates of the particles of continuum $K$ at some fixed time $t_{0}$. The spatial coordinates $x_{i}$ for any particle $a_{i}^{K}$ at any time $t, t \geq t_{0}$, are then assumed to be given by the functions* $x_{i}^{*}\left(a_{i}^{K}, t\right)$ which are taken to be single-valued and at least twice continuously differentiable with respect to each of their variables:

$$
x_{i}=x_{i}^{*}\left(a_{i}^{K}, t\right), \quad t \geq t_{0},
$$

and $a_{i}^{K} \equiv x_{i}^{*^{K}}\left(a_{i}^{K}, t_{0}\right)$. The transformations are assumed to be one-to-one, so that the inverse transformations $a_{i}^{K}\left(x_{i}, t\right)$ also exist and are twice continuously differentiable. The flow velocities or "particle velocities" for continuum $K, v_{i}^{*^{K}}\left(a_{i}^{K}, t\right)$, are then defined as:

$$
v_{i}^{* K}\left(a_{i}^{K}, t\right) \equiv \frac{\partial x_{i}^{* K}}{\partial t}=v_{i}^{K}\left(x_{i}, t\right),
$$

where the $v_{i}^{K}$ are defined by the inverse transformation. Similarly, the Jacobian of Eq. (A-1) is given as $\Delta^{* \pi}$ or $\Delta^{K}$ :

$$
\Delta^{* K}\left(a_{i}^{K}, t\right) \equiv \operatorname{det}\left\{\frac{\partial x_{i}^{* K}}{\partial a_{i}^{K}}\right\}=\Delta^{K}\left(x_{i}, t\right) .
$$

If the integral of Eq. (3) is changed with the use of Eq. (A-1) to an integration at time $t=t_{0}$ over the volume $\tau_{0}^{K}$, then

$$
A^{K}(t)=\int_{\tau_{0} x^{K}} \alpha^{*^{K}}\left(a_{i}^{K}, t\right) \Delta^{*^{K}} d \tau_{0}^{K} .
$$

The definition of the time derivative given in Eq. (5) is therefore equivalent to

$$
\frac{d A^{K}}{d t}=\int_{\tau_{0} K}\left(\frac{\partial \alpha^{* K}}{\partial t} \Delta^{*^{K}}+\alpha^{*^{K}} \frac{\partial \Delta^{* K}}{\partial t}\right) d \tau_{0}^{K} .
$$

But it is readily shown that ${ }^{7}$

$$
\frac{\partial \Delta^{* K}}{\partial t}=\frac{\partial v_{i}^{K}}{\partial x_{i}} \Delta^{* K} \equiv v_{i, i}^{K} \Delta^{* K}
$$

and, therefore, Eq (A-4) may be written as

$$
\frac{d A^{K}}{d t}=\int_{\tau_{0} K}\left(\frac{\partial \alpha^{* K}}{\partial t}+v_{i, i}^{K} \alpha^{*^{K}}\right) \Delta^{*^{K}} d \tau_{0}^{K} .
$$

Transformation of Eq. (A-5) to spatial coordinates leads to Eq. (6).

*In this Appendix only, an asterisk on any function indicates that its variables are $a_{i}, t$; functions without asterisks have the independent variables $x_{i}, t$.

'See [5], Eq. (7.07). 


\section{REFERENCES}

1. H. W. Liepmann and A. E. Puckett, Introduction to aerodynamics of a compressible fluid, John Wiley and Sons, New York, 1947, chap. 7

2. S. Goldstein, Modern developments in fluid dynamics, vol. I, Clarendon Press, Oxford, 1938, pp. 95-105

3. L. Prandtl, Essentials of fluid dynamics, Blackie and Sons, Ltd., Glasgow, 1952, chap. II etc.

4. S. Chapman and T. G. Cowling, The mathematical theory of non-uniform gases, Cambridge University Press, Cambridge, 1953, chap. 3

5. J. O. Hirschfelder, C. F. Curtiss and R. B. Bird, Molecular theory of gases and liquids, John Wiley and Sons, New York, 1954, chap. 7

6. Th. von Kármán, Sorbonne Lectures, Paris, France, 1950-51

7. S. S. Penner, Introduction to the study of chemical reactions in flow systems, Butterworths' Scientific Publications, London, 1955, chap. 2

8. For related treatments, see the following:

J. Stefan, Sitzber, Akad, Wiss. Wien 632, 63 (1871);

C. Eckart, Phys. Rev. 58, 267 (1940);

C. Truesdell, J. Rational Mech. Anal. 1, 125 (1952)

9. R. Courant and K. O. Friedrichs, Supersonic flow and shock waves, Interscience Publishers, New York, 1948, Sec. 7 